\title{
Characterization of Sub-THz Photonic-Transmitters Based on GaAs-AlGaAs Uni-Traveling-Carrier Photodiodes and Substrate-Removed Broadband Antennas for Impulse-Radio Communication
}

\author{
Yu-Tai Li, J.-W. Shi, Cheng-Yu Huang, Nan-Wei Chen, Shu-Han Chen, J.-I. Chyi, and Ci-Ling Pan
}

\begin{abstract}
A novel photonic transmitter for wireless terahertz (THz) impulse-radio (IR) communication is realized by monolithic integration of a GaAs-AIGaAs-based uni-traveling-carrier (UTC) photodiode with a substrate-removed broadband antenna. The device can radiate strong sub-THz pulses $(20-\mathrm{mW}$ peak-power) with a narrow pulsewidth $(<2 \mathrm{ps})$ and wide bandwidth $(100 \sim 250 \mathrm{GHz})$. The maximum average power emitted by our device, under the same $\mathbf{T H z}$ time-domain spectroscopic setup, is around ten times higher than that of the low-temperature-grown GaAs-based photoconductive antenna, while with a much lower dc bias ( 9 versus $35 \mathrm{~V}$ ). The bias-dependent peak output powers of our devices suggest their suitability for application as a data modulator/emitter for photonic THz IR communication.
\end{abstract}

Index Terms-Photonic transmitter (PT), submillimeter-wave, terahertz (THz), uni-traveling-carrier photodiode (UTC-PD).

\section{INTRODUCTION}

$\mathbf{R}$ ECENTLY, the demand for low-transmission-power high-bit-rate wireless local area networks in homes and offices has grown rapidly because of the increase in data volume that is being transmitted between various portable devices. Impulse radio (IR) is one technology that has been proposed to meet this demand. Admitting that, there remains a considerable challenge [1]. For IR applications, the approach of using short (sub-picoseconds) electrical pulses directly generated by electronic devices such as nonlinear transmission lines [2] often suffers from huge propagation loss in free-space. The use of photonic technology in an IR system, where the optical pulse signal is distributed through a low-loss optical fiber and an optical-pulse triggered photoconductive (PC) antenna or photonic transmitter (PT) to radiate the converted electrical signal to the user-end, is an interesting alternative. Some sub-terahertz (sub-THz) signal communication links have

\footnotetext{
Manuscript received March 6, 2008; revised May 1, 2008. This work was supported in part by the National Science Council (NSC) of Taiwan through various grants including PPAEU-II, and in part by the Ministry of Education's ATU program, Taiwan.

Y.-T. Li and C.-L. Pan are with the Department of Photonics, National Chiao Tung University, Hsinchu, Taiwan, R.O.C.

J.-W. Shi, C.-Y. Huang, N.-W. Chen, S.-H. Chen, and J.-I. Chyi are with the Department of Electrical Engineering, National Central University, Taoyuan, Taiwan, R.O.C. (e-mail: jwshi@ee.ncu.edu.tw; clpan@ faculty.nctu.edu.tw).

Color versions of one or more of the figures in this letter are available online at http://ieeexplore.ieee.org.

Digital Object Identifier 10.1109/LPT.2008.926855
}

already been demonstrated [3], [4]. Low-temperature-grown GaAs (LTG-GaAs)-based photodetectors, excited at $800 \mathrm{~nm}$, have also been shown to be a promising choice for use in PT or $\mathrm{PC}$ technology for the generation of continuous-wave $\mathrm{THz}$ signals or THz pulses [5], [6]. Nevertheless, the external efficiency of LTG-GaAs-based photodiodes (PDs) is still lower than that of the typical GaAs-based p-i-n PDs, because of the existence of recombination centers in the photo-absorption layer [6].

Another attractive choice for PT applications is the UTC-PD [7]. Recently, we have reported a high-speed GaAs-AlGaAs-based UTC-PD [8], composed of a GaAs-based p-type photo-absorption layer and an $\mathrm{Al}_{0.15} \mathrm{Ga}_{0.85}$ As-based collector layer. In this study, we analyze the detailed power and bias dependent behaviors of a sub-THz PT [9], fabricated by monolithically integrating an edge-coupled GaAs-AlGaAs-based UTC-PD [8] and a substrate-removed circular disk monopole antenna [10]. Removal of the substrate eliminates the need for the integration of an additional silicon lens with the PT [6], [11]. The dynamic behavior of this device is characterized by using a $\mathrm{THz}$ time-domain spectroscopic (TDS) system [4]. Under femtosecond optical pulse excitation, our device radiates strong sub-THz pulses with around $20-\mathrm{mW}$ peak-power and 2-ps pulsewidth. The average radiated power is as high as ten times that of a typical LTG-GaAs-based PC antenna (LTG-PC) [12], while with a much lower dc bias (9 versus $35 \mathrm{~V})$.

\section{DeVice Structure}

Fig. 1 shows the top-view of the sub-THz PT [9]. The simulated frequency response of the microwave reflection parameter $\left(S_{11}\right)$ for the antenna is shown in Fig. 1, inset (a). Using the $-10-\mathrm{dB}$ line on the $\mathrm{y}$-axis as the reference [13], we can clearly see that most of the frequency component $(100-350 \mathrm{GHz})$ in the simulated frequency band has a low reflection loss $(<-10 \mathrm{~dB})$. This indicates the good broadband radiation characteristics of our antenna. Inset (b) in Fig. 1 shows a conceptual band diagram of the UTC-PD. Our UTC-PD has a higher breakdown voltage and more significant bandwidth enhancement effect under a much lower output photocurrent density than that of the traditional InP-based UTC-PD, due to the larger bandgap of the collector and the larger slope of graded p-type doping profile in the absorption layer [8]. Instead of using an additional Si-lens for efficient collection of emitted $\mathrm{THz}$ power, we remove part of the GaAs substrate from just below the circular disk of the antenna by selective wet etching and back-side lithography [6]. 
(a)

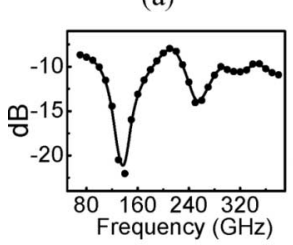

(b)
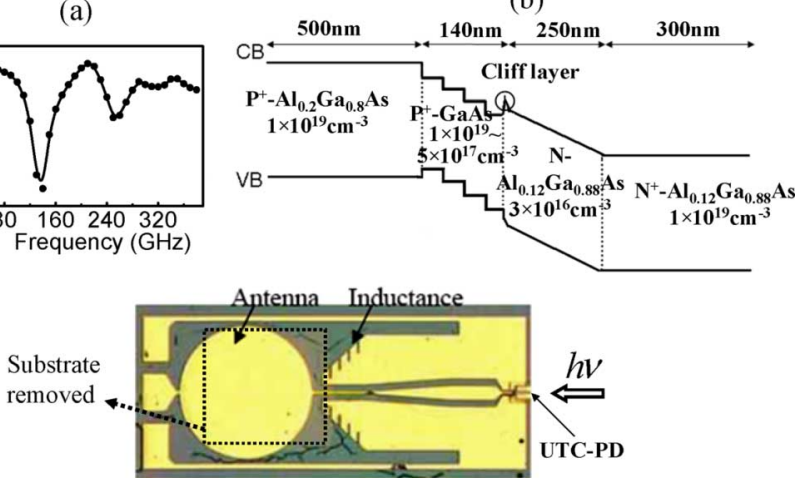

Fig. 1. Top-view of the sub-THz PT device. Inset (a) shows the simulated frequency response of the designed antenna. Inset (b) shows a conceptual band diagram of the demonstrated UTC-PD.

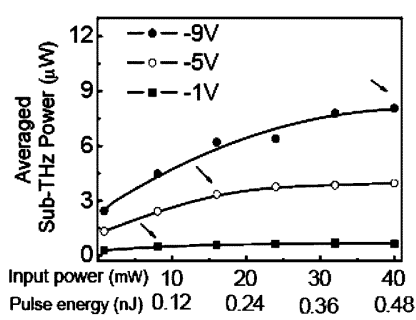

(a)

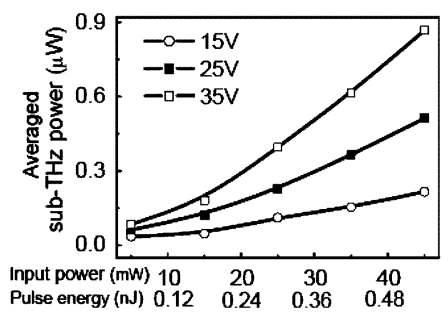

(b)
Fig. 2. Measured sub-THz power versus the injected optical power of (a) our device and (b) an LTG-PC under different reverse biases.

The thin membrane disk has a thickness of around $5 \mu \mathrm{m}$. Its corresponding cut-off frequency $(\sim 4 \mathrm{THz})$ of substrate-mode is thus much higher than the designed frequency band $(100 \sim$ $300 \mathrm{GHz}$ ) of the antenna. The substrate-mode problem [11] can thus be overcome and the radiation power can also be improved.

\section{EXPERIMENTAL METHODS AND RESULTS}

We tested the performance by directly exciting the device with a femtosecond mode-locked Ti : sapphire laser. The active length and width of the measured device were 14 and $2 \mu \mathrm{m}$, respectively, and the total active area, which includes the area of the pedestal for the pad connection, was around $64 \mu \mathrm{m}^{2}$. The radiated power was first measured by a liquid-Helium-cooled Si bolometer, which was carefully calibrated to the blackbody source. We also measured and calibrated the radiated $\mathrm{THz}$ beam propagation loss in air by measuring the $\mathrm{THz}$ intensity as a function of the propagation distance. At a relative humidity of $\sim 40 \%$, the propagation loss was about $0.082 \mathrm{~cm}^{-1}$.

Fig. 2(a) shows the measured sub-THz power versus injected optical power under different reverse bias voltages. The collected power in our measurement system was corrected for a propagation loss of around $4.8 \mathrm{~dB}$ after $12 \mathrm{~cm}$ of propagation. It is obvious that the values for saturated injected optical pulse energy, indicated by the arrow heads in Fig. 2(a), increased with the absolute value of the reverse bias voltage. This phenomenon may be similar to the saturation mechanism reported for the InP-InGaAs-based UTC-PDs [14], where the electron-induced space-charge screening effect in the collector layers can be minimized by increasing the external electrical field in this layer. The measured sub-THz power versus optical pumping power under

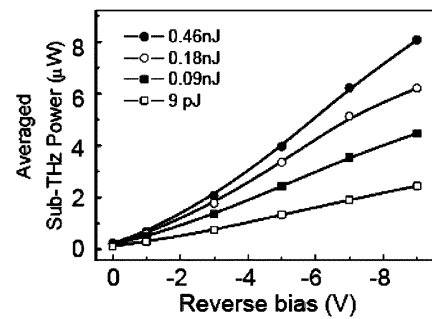

(a) (b)

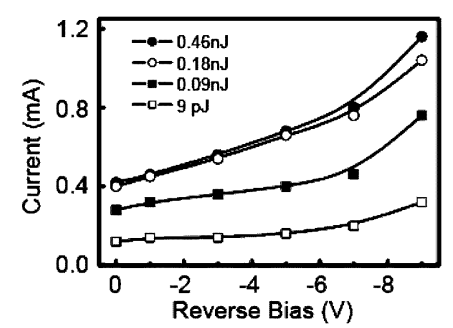

Fig. 3. (a) Bias dependent sub-THz power and (b) bias dependent photocurrent under different power excitation.

different bias voltages for our LTG-PC is shown in Fig. 2(b) for comparison. The values are close to those reported for an LTG-PC with an antenna of the same design [12].

Fig. 3(a) shows a plot of the average emitted sub-THz power under different optical pumping pulse energies as a function of bias voltage. When the reverse bias is less than around $-5 \mathrm{~V}$, the radiated sub-THz power exhibits an ideally quadric relation with the reverse bias voltage. This is due to the linear dependence of the output photocurrent on the reverse bias voltage $(<-5 \mathrm{~V})$, as shown in Fig. 3(b). When the reverse bias voltage increases further, however, the output sub- $\mathrm{THz}$ power exhibits a linear dependence on the bias voltage. This can be attributed to the fact that the value of the external applied electrical field $\left(\sim 3.6 \times 10^{5} \mathrm{~V} / \mathrm{cm}\right)$ in the $\mathrm{Al}_{0.12} \mathrm{Ga}_{0.88}$ As-based collector layer is close to its breakdown field. Thus, the avalanche-induced bandwidth degradation phenomenon of the PD limits the superlinear increase of output power versus the bias. Fig. 3(b) clearly shows that the measured photocurrent increases superlinearly with the bias voltage when it is over $-5 \mathrm{~V}$. This indicates that the avalanche phenomenon really does occur. The maximum measured output sub-THz average power of our device, under the highest reverse bias and optical pumping pulse energy $(0.48 \mathrm{~nJ})$, is around $8 \mu \mathrm{W}$.

We utilized a THz-TDS system [4] to investigate the waveform of the radiated sub- $\mathrm{THz}$ impulse that could be applied to the IR communication system. The same mode-locked Ti : sapphire laser was used to pump our device and the injected optical pulse energy is $\sim 480 \mathrm{pJ} / \mathrm{pulse}$. The radiated signal was then probed with a PC dipole antenna fabricated on LTG-GaAs and integrated with a silicon lens [12]. With identical PC antenna as the emitter and receiver, the THz-TDS system exhibits a bandwidth exceeding $2 \mathrm{THz}$ and signal-to-noise ratio better than 1000. Fig. 4(a) shows waveforms of the radiated sub-THz pulses by the device under different reverse biases. The corresponding spectrum for a bias of $(-5 \mathrm{~V})$ is shown in Fig. 4(b).

The peak-power (square of the peak electrical field) of each trace changes significantly with the bias. As the bias voltage sweeps from -1 to $-5 \mathrm{~V}$, the peak-power increases about ten times. This result is an indication of the potential of this device to serve as a sub-THz emitter and data modulator, using the bias modulation technique [15]. The full-width at half-maximum (FWHM) of the main peak of the waveform is around $1.8 \mathrm{ps}$ and bandwidth of the corresponding spectrum covers from $100 \mathrm{GHz}$ to around $250 \mathrm{GHz}$. This is somewhat smaller but comparable to that by the simulation result $(100-350 \mathrm{GHz})$ of 


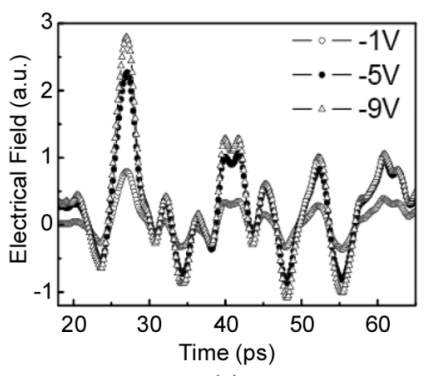

(a)

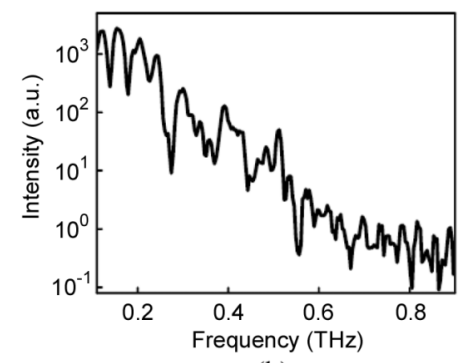

(b)
Fig. 4. (a) Bias dependent waveforms of the radiated sub-THz pulses and (b) power spectrum of radiated sub- $\mathrm{THz}$ pulse for a bias of $(-5 \mathrm{~V})$.

the antenna, as shown in the inset to Fig. 1. The bandwidth limitation may be attributed to the serious high-frequency roll-off of the active UTC-PD when the operating frequency exceeded $300 \mathrm{GHz}$.

From the bias-dependent waveforms of the radiated sub- $\mathrm{THz}$ pulses shown in Fig. 4(b), we can clearly see that the shapes of the measured impulse response do not change with the bias, exhibiting the same FWHM ( $\sim 1.8 \mathrm{ps})$. The phenomenon of ringing in these measured waveforms is consistent with our finite-difference time-domain simulation result using SEMCAD (Schmid \& Partner Engn. AG, Zurich, Switzerland). During our simulation, we assumed that the excited electrical pulse covers the frequencies ranging from 100 to $300 \mathrm{GHz}$ and a long ringing exists in the radiated waveform. Such phenomenon is also reported for other PTs [16] and can be ascribed to the fact that some of the frequency components of the impulse response generated from the UTC-PD have been truncated by the frequency selective antenna. In addition, the multiple reflections between substrate-removed antenna and microwave probe may also cause such phenomenon [16].

We have roughly estimated the highest radiated peak-power and peak-current density from the radiated pulse waveforms, average power, and photocurrent which could be as high as $20 \mathrm{~mW}$ and $\sim 14 \mathrm{MA} / \mathrm{cm}^{2}$, respectively. The peak power radiated by the present device is five times larger than that reported previously for a STR-PD-based PT with a narrowband resonant slot antenna [6]. Furthermore, our device could sustain a much higher reverse bias voltage ( -9 versus $-4 \mathrm{~V})$, much higher optical pulse energy illumination (480 versus $3.8 \mathrm{pJ} /$ pulse), and over ten times higher peak-output-current density $\left(\sim 14 \mathrm{MA} / \mathrm{cm}^{2}\right.$ versus $\left.380 \mathrm{kA} / \mathrm{cm}^{2}\right)$ without failure than that reported for a InP-InGaAs-based UTC-PD [14] with a similar collector layer thickness $(\sim 250 \mathrm{~nm})$. We note also that the LTG-GaAs-based PC antenna biased at $9 \mathrm{~V}$ can only deliver around $1 / 10$ of the maximum average power $(0.9$ versus $8 \mu \mathrm{W})$ under the same measurement setup, for a much higher bias voltage (35 versus $9 \mathrm{~V})$.

\section{CONCLUSION}

We have studied the dynamic behavior of a PT constructed by monolithically integrating a GaAs-AlGaAs-based UTC-PD with a substrate-removed monopole antenna. The PT radiated sub-THz pulses less than $2 \mathrm{ps}$ in width, with peak power as high as $20 \mathrm{~mW}$ and a wide bandwidth (beyond $250 \mathrm{GHz}$ ). The bias dependent high-peak-power performance of our device suggests its suitability for application as a photonic emitter and possibly as a data modulator in sub-THz IR communication systems.

\section{REFERENCES}

[1] M. Z. Win and R. A. Scholtz, "Impulse radio: How it works," IEEE Commun. Lett., vol. 2, no. 2, pp. 36-38, Feb. 1998.

[2] M. J. W. Rodwell, S. T. Allen, R. Y. Yu, M. G. Case, U. Bhattacharya, M. Reddy, E. Carman, M. Kamegawa, Y. Konishi, J. Pusl, and R. Pullela, "Active and nonlinear wave propagation devices in ultrafast electronics and optoelectronics," Proc. IEEE, vol. 82, no. 7, pp. 1037-1059, Jul. 1994.

[3] H. Togo, P.-C. P. Sah, N. Shimizu, and T. Nagatsuma, "Gigabit impulse radio link using photonic signal-generation techniques," in Eur. Microwave Conf. 2005, Oct. 2005, vol. 1, pp. 4-7.

[4] T.-A. Liu, G.-R. Lin, Y.-C. Chang, and C.-L. Pan, "Wireless audio and burst communication link with directly modulated $\mathrm{THz}$ photoconductive antenna," Opt. Express, vol. 13, no. 25, pp. 10416-10423, Dec. 2005 .

[5] S. M. Duffy, S. Verghese, K. A. McIntosh, A. Jackson, A. C. Gossard, and S. Matsuura, "Accurate modeling of dual dipole and slot elements used with photomixers for coherent terahertz output power," IEEE Trans. Microw. Theory Tech., vol. 49, no. 6, pp. 1032-1038, Jun. 2001.

[6] Y.-T. Li, J.-W. Shi, C.-L. Pan, C.-H. Chiu, W.-S. Liu, N.-W. Chen, C.-K. Sun, and J.-I. Chyi, "Sub-THz photonic-transmitters based on separated-transport-recombination photodiodes and a micromachined slot antenna," IEEE Photon. Technol. Lett., vol. 19, no. 11, pp. 840-842, Jun. 1, 2007.

[7] H. Ito, T. Furuta, F. Nakajima, K. Yoshino, and T. Ishibashi, "Photonic generation of continuous $\mathrm{THz}$ wave using uni-traveling-carrier photodiode," J. Lightw. Technol., vol. 23, no. 12, pp. 4016-4021, Dec. 2005.

[8] J.-W. Shi, Y.-T. Li, C.-L. Pan, M. L. Lin, Y. S. Wu, W. S. Liu, and J.-I. Chyi, "Bandwidth enhancement phenomenon of a high-speed GaAs-AlGaAs based unitraveling carrier photodiode with an optimally designed absorption layer at an $830 \mathrm{~nm}$ wavelength," Appl. Phys. Lett., vol. 89, no. 5, p. 053512, 2006.

[9] Y.-T. Li, C.-L. Pan, J.-W. Shi, C.-Y. Huang, N.-W. Chen, S.-H. Chen, and J.-I. Chyi, "Sub-THz photonic-transmitters based on GaAs/AlGaAs uni-traveling carrier photodiode and micromachined circular disk monopole antenna for ultra-wideband communication," in Proc. Conf. Lasers Electro-Opt. (CLEO 2008), San Jose, CA, May 2008, Paper CThD5.

[10] Y.-C. Liang and N.-W. Chen, "An ultra-broadband coplanar waveguide-fed circular monopole antenna," in Proc. EuCAP 2007, Edinburgh, U.K., Nov. 2007.

[11] G. M. Rebeiz, "Millimeter-wave and terahertz integrated circuit antennas," Proc. IEEE, vol. 80, no. 11, pp. 1748-1770, Nov. 1992.

[12] M. Tani, S. Matsuura, K. Sakai, and S. Nakashima, "Emission characteristics of photoconductive antennas based on low-temperature-grown GaAs and semi-insulating GaAs," Appl. Opt., vol. 36, pp. 7853-7859, Oct. 1997.

[13] W. L. Stutzman and G. A. Thiele, Antenna Theory and Design, 2nd ed. Hoboken, NJ: Wiely, 1998, ch. 6.

[14] N. Shimizu, N. Watanabe, T. Furuta, and T. Ishibashi, "InP-InGaAs uni-traveling-carrier photodiode with improved 3-dB bandwidth of over 150 GHz," IEEE Photon. Technol. Lett., vol. 10, no. 3, pp. 412-414, Mar. 1998.

[15] A. Hirata, T. Furuta, H. Ito, and T. Nagatsuma, "10-Gb/s millimeterwave signal generation using photodiode bias modulation," J. Lightw. Technol., vol. 24, no. 4, pp. 1725-1731, Apr. 2006.

[16] H. Togo, A. Sasaki, A. Hirata, and T. Nagatsuma, "Characterization of millimeter-wave antenna using photonic measurement techniques," RF Microwave Computer-Aided Eng., vol. 14, no. 3, pp. 290-297, Apr. 2004. 